\title{
Análisis del desempeño virtual del rol de estudiantes de Ecacen, desde la perspectiva del desempeño del tutor como mediador del proceso
}

Fabián José Mendoza Stave ${ }^{1}$

\begin{abstract}
Resumen
El objetivo es analizar el desempeño virtual de los estudiantes de la Escuela de Ciencias Administrativas, Económicas, Contables y de Negocios (Ecacen) frente a su rol como estudiantes, desde la perspectiva del desempeño del tutor como mediador del proceso. La técnica de recolección de información utilizada fue la encuesta como técnica de recogida de datos cuantitativos. Ella permitirá una descripción estructurada y general de las variables consideradas. Se diseñó una encuesta para los estudiantes para obtener información sobre los siguientes aspectos: calidad, desempeño, éticas y valores, ambientes virtuales de aprendizaje, mediaciones pedagógicas.
\end{abstract}

Palabras clave: TIC, Aprendizaje autónomo, tutor, virtual, E-learning, desempeño. 


\title{
An analysis of ECACEN students' virtual performance, from the perspective of their teaching mentor's performance as a mediator in the process
}

\begin{abstract}
This research is linked to the distance learning process and the relationship between the student and the tutor, because in many parts of the world this methodology it is not truthful at all, by their style to work. However if you are honest, responsible and willingness to do, it is an entirely welcoming model. The aim is to analyze the ECACEN students' performance face to their virtual role, from the tutor's perspective as a mediator in the process. The data collection technique used was the survey as a technique for collecting quantitative data, allowing a structured and comprehensive description of the variables considered. We designed a survey for students to learn about the following aspects: quality, performance, ethics and values, virtual learning environments, pedagogical mediations.
\end{abstract}

Keywords: Tics, autonomous learning, tutor, virtual, E-learning, performance

Recibido: 22 Marzo de 2011 Aceptado: 1 Junio de 2011

\section{lntroducción}

La utilización de la tecnología de la información y comunicación como herramienta para la educación, en este caso a distancia, han tenido una rápida expansión. No obstante, aún no hay suficiente refulgencia entre los docentes y estudiantes sobre la utilidad de la misma en el ámbito educativo, como cuáles son las aplicaciones propias de la informática, cuáles son sus usos como herramienta para el trabajo intelectual, en qué áreas del conocimiento es fructuosa su utilización, qué requerimientos educativos previos deben de poseer los involucrados, dentro de qué marco de política de desarrollo nacional se inserta. Sin embargo, prosperar en esta estrategia demanda de un contexto pedagógico participativo, individualizado tanto del tutor como del alumno y el personal involucrado; elección de las herramientas, tecnologías, estrategia, 
acciones y plataformas, adecuándose a las características oportunas del trabajo educativo como a las diversas circunstancias de las instituciones.

En este sentido, se recreará una situación educativa centrada en el alumno a partir de la estimulación de su autoaprendizaje y el desarrollo de su pensamiento crítico y creativo mediante el empleo de tecnología de realidad virtual; respondiendo a la necesidad y exigencia de diversificar y flexibilizar las oportunidades de aprender cualquier área de conocimiento propias de su rama de servicio en espacio, tiempo y de manera distinta, atendiendo a las necesidades institucionales, de grupo e individuales.

Los pensamientos de Vygostki con relación al docente, como mediador y su función fundamentalmente problematizadora, son ejemplos bien conocidos en la historia de la educación que nos muestran que las competencias comunicativas no son un nuevo requerimiento hacia la docencia, han estado presentes, como parte de un paradigma axiológico sobre lo educativo, desde la antigüedad y en diversos contextos civilizatorios.

Desde hace décadas se ha venido instruyendo y formando al estudiante bajo una metodología convencional presencial buscando cada día formar al educando de una manera integral. Educación Presencial se refiere a que tienes que tomar el curso en algún aula. Los estudiosos del tema educativo se dieron cuenta en que había que cambiar y esto representó un momento exacto para la aplicación pedagógica de la técnica y equipos, el establecimiento de un nuevo proceso capaz de innovar los procedimientos educativos, dada su indudable efectividad para comunicar conocimientos, manifestar habilidades y destrezas y contribuir a internalizar los valores democráticos, surgió entonces el aprendizaje significativo y en su momento el instruccional.

Una vez entrado al modelo de educación a distancia, se separa también a utilizar medios y mediaciones acordes y necesarias con este modelo, de tal forma que se creen mecanismos que ayuden al proceso de formación y de aprendizaje de un estudiante. Pero, desde luego, esta investigación está ligada más bien al proceso de educación a distancia como tal y el rol entre ese estudiante y el tutor, porque en muchas partes del mundo está en entredicho este tipo de metodología, por la forma de trabajar pero, si se cuenta con honestidad, responsabilidad y disposición para hacerlo, es un modelo totalmente acogedor. La educación a distancia se ha convertido en la mejor elección para quienes desean estudiar sin necesidad de hacer grandes gastos de tiempo y dinero en desplazamientos y presencia en aulas de clase. A diferencia de la educación normal, en la actualidad los estudiantes reciben los contenidos de las asignaturas a través de Internet, televisión por cable, video conferencia y otros medios tecnológicos.

Cuando existe entonces educación a distancia, entre otros actores importantes se encuentra el tutor y el estudiantes, estos últimos como grandes ejecutores 
de este proceso y que la calidad de la educación, independientemente de que metodología utiliza, tanto en la educación presencial como en la virtual, ambos son piezas fundamentales para el fortalecimiento del proceso. He aquí, entonces, que ambas metodologías son homólogas en procesos de calidad porque casi todo los procesos depende de estos actores principales. Aunque existan otros, cada una tiene sus cartas a tomar para cualquier paso.

La Universidad Nacional Abierta y a Distancia (UNAD) tiene su propio campus, el cual tiene como propósito general garantizar la sostenibilidad y consolidación de la plataforma de Campus Virtual como escenario básico para el desarrollo de procesos de aprendizaje autónomo y la formación a distancia basados en tecnologías digitales y telemáticas, contribuyendo de manera específica al cumplimiento de la misión institucional y de sus responsabilidades formativas, investigativas y de proyección social. ${ }^{2}$ De tal manera, entonces, la UNAD, a través de su campus, utiliza una estrategia de mediación para cumplir con su proceso de aprendizaje y no como algunos foros en la Red que dicen: el fracaso del proceso virtual de UNAD, escrito por algún inconforme de los procesos autónomos de aprendizaje. Sin embargo, hay que ser enfático: como en todo proceso, hay fallas y la idea e s aprender de los errores y estos con el tiempo van quedando atrás, por que se solucionan.

Entrando en materia, el rol del tutor virtual debe fraccionar con cualquier representación que ya haya sido creado por la presencialidad debido a que debe ir mucho más lejos en crear conciencia en los estudiantes de la importancia que tiene el autoaprendizaje y, más que esto, instruir a pensar y a utilizar de manera adecuada las herramientas tecnológicas que en la actualidad se manejan, las TIC. Debe ser una persona en persistente crecimiento, con alta capacidad investigativa, líder, facilitador con un enérgico compromiso con la sociedad y el impacto benévolo que puede crear en ella. Debe ser un firme motivador, consciente de que la capacitación y el perfeccionamiento continuo debe ser cultura de vida para él y para los influidos. Cosa que aparentemente no se está dando.

En el proceso educativo siempre se encontrará estudiantes y tutores. Esta relación debe estar planteadas por funciones y perfiles claros que permitan establecer el papel que cada uno juega en el proceso de aprendizaje. El tutor está enfocado en el proceso de aprendizaje donde puede guiar, acompañar, y asesorar para que pueda apropiarse del conocimiento utilizando como herramienta; es quien analizar, sintetizar, inferir, cuestionar, reflexionar desde proceso cognitivo pero a la vez humanista; parte de la innovación haciendo uso de los múltiples recurso de las TIC que puedan brindar beneficio a sus educandos y a la educación virtual. $\mathrm{Su}$ fin es próximo es generar una interacción permanente entre los participantes apelando a recursos innovadores como talleres virtuales, foros de tipo social (cibercafé, tablón de anuncios), 
intercambio de experiencias, etc. Por su parte, el estudiante virtual tiene su mente abierta para compartir cuestiones personales sobre sus vidas, trabajos y otras experiencias educativas. Esta es una condición importante para la construcción de comunidades virtuales. Tiene la capacidad de automotivarse y autodisciplinarse, porque la flexibilidad en la manera de impartir los cursos requiere responsabilidad y compromiso, puede trabajar de manera colaborativa, identifica la importancia de pensamiento crítico en el proceso, descubre rápidamente que el tutor actúa como facilitador y por tanto cada participante es consciente de que debe hacerse cargo del proceso por sí mismo, lo cual implica, asumir un rol en el que se evitan las creencias y prejuicios y se cuestionan los supuestos que aparecen.

Teniendo los roles de ambos actores principales del proceso, la idea de esta investigación es observar si cada uno de ellos lo hace correctamente, para ello entraremos a recolectar la información de manera prudente y relevante para dar buenos resultados. En este caso solo se diseñará una encuesta a los estudiantes de los cuales se propondrá y servirá de ejercicio para otra investigación, dependiendo de los resultados obtenidos.

Los objetivos del estudio son:

- Analizar el desempeño virtual de los estudiantes de Ecacen frente a su rol como estudiantes, desde la perspectiva del desempeño del tutor como mediador del proceso.

- Identificar si la educación que se da a través de mediaciones tecnológicas en la educación a distancia satisface necesidades formativas e informativas.

- Concientizar el uso de la información como proceso honesto y responsable por parte de aprendiente.

- Determinar a el grado de acompañamiento del tutor en el proceso de aprendizaje.

- Analizar si se reproducen esquemas tradicionales de educación: de reproducción y consumo informativo pero utilizando las más modernas tecnologías.

\section{Metodologia}

Esta investigación se realizó a través de un proceso sistémico con un enfoque cualitativo y cuantitativo. La investigación es de tipo no experimental, se 
observó lo investigado y se digitó como se presentaron los resultados. El tipo de estudio fue descriptivo-analítico, describiendo cómo se presenta el desempeño de los actores. El enfoque cuantitativo permitió verificar el cumplimiento de algunas características expuestas, representadas en valores numéricos para hacer un análisis estadístico. El enfoque cualitativo describió cada una de las situaciones expuestas teniendo en cuenta el desempeño y perspectiva de los encuestados.

\section{Sujetos participantes}

La población objeto de estudio es el colectivo de todos los alumnos de los cursos de Gerencia Integral, Administración Pública y Geografía Económica. Se realizó una muestra, la previsión de la población eran unos 520 alumnos en Geografía Económica, 760 alumnos en Administración Pública y 154 alumnos en Gerencia Integral, para un total de 1.360 estudiantes, de todos se toma una muestra de que garantizaban un nivel de confianza del 95\% y un error muestral de $\pm 5 \%$. Sin embargo se encuestaron 350 estudiantes.

\section{Cálculo de la muestra}

$$
n=\frac{N z^{2} \alpha / 2 P(1-P 9}{(N-1) e^{2}+z^{2} \alpha / 2 P(1-P 9}
$$

donde $\mathbf{z} \boldsymbol{\alpha} / \mathbf{2}$

$$
\begin{aligned}
& \text { z: correspondiente al nivel de confianza elegido } \\
& \text { P: proporción de una categoría de la variable } \\
& \text { e: error máximo } \\
& \text { N: tamaño de la población } \\
& \text { Nivel de confianza de } \quad 95 \% \\
& \text { z= } \quad 1,96 \\
& \mathbf{P}=0,50 \quad \mathbf{n}=\mathbf{3 0 0} \\
& \mathbf{N}=1360 \\
& \mathbf{e}=0,05
\end{aligned}
$$

\section{Variables e instrumentos}

\section{a) Encuestas}

La encuesta como técnica de recolección de datos cuantitativos permitirá una descripción estructurada y general de las variables consideradas. Se pretende obtener información sobre los siguientes aspectos: Calidad, desempeño, éticas y valores, ambientes virtuales de aprendizaje, mediaciones pedagógicas.

Fuente de las encuestas: Cursos académicos de Administración pública, gerencia integral y geografía económica de la escuela ECACEN - UNAD. 


\section{Revista de}

\section{Resultados de la investigación}

La figura 1 muestra los porcentajes en intervalos que corresponden a periodos académicos matriculados por los estudiantes

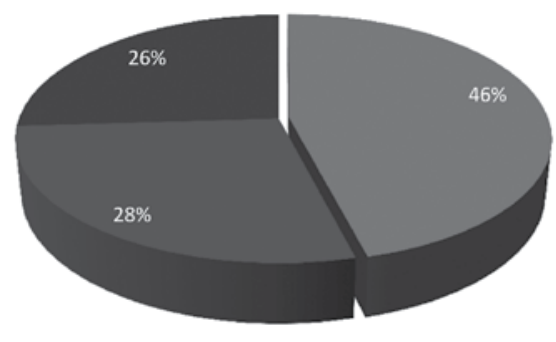

\begin{tabular}{|l|l|}
\hline Entre 2 a 4 & $46 \%$ \\
\hline Entre 5 a 7 & $28 \%$ \\
\hline Entre 8 a 10 & $26 \%$ \\
\hline
\end{tabular}

Figura 1. El $46 \%$ de los estudiantes está en periodos entre 8 y 10 , el $28 \%$ se encuentra entre 5 y 7 periodos y solo el $26 \%$ se encuentra de 2 a 4 periodos, corroborando así que gran porcentaje de los estudiantes se encuentra en periodos avanzados y de los cuales tienen un recorrido mayor en la universidad.

La figura 2 muestra los porcentajes sobre el motivo si ha desarrollado actividades sin estar debidamente preparado (sin leer) y ha asumido conductas inadecuadas por ejemplo, ¿cómo abrir el texto?

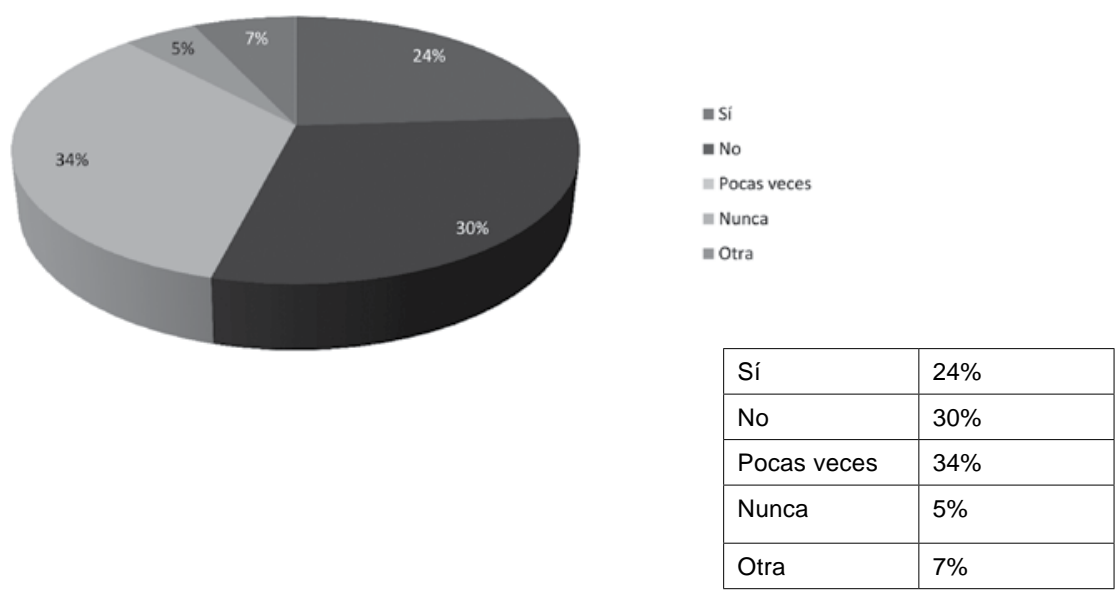

Figura 2. El $34 \%$ de los encuestados comenta que pocas veces entran a realizar la actividad sin haber leído, el $24 \%$ dice que si entra sin haber leído el modulo, sin embargo el $30 \%$ dice que honestamente no lo ha hecho nunca, pero el $7 \%$ dice que lo hace por apoyo educativo o porque el sistema lo permite. 


\section{Fabián José Mendoza Stave}

Análisis del desempeño virtual del rol de estudiantes de Ecacen, desde la perspectiva del desempeño del tutor como mediador del proceso, artículo producto de la investigación

La figura 3 muestra los porcentajes sobre la creencia de que los ambientes virtuales pueden fomentar la conducta no ética académica en estudiantes.

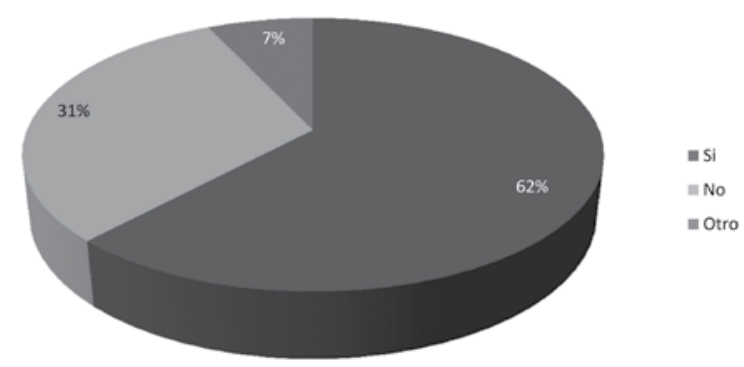

\begin{tabular}{|l|l|}
\hline $\mathrm{Si}$ & $62 \%$ \\
\hline No & $31 \%$ \\
\hline Otro & $7 \%$ \\
\hline
\end{tabular}

Figura 3. El $62 \%$ dice que los ambientes virtuales de aprendizaje fomenta conductas no éticas, el $31 \%$ comenta que no, sin embargo el $7 \%$ afirma que cada persona es consciente de su conducta y que si el actúa de esa manera sabe que es un error, la ética de la convicción.

La figura 4 muestra los porcentajes sobre las características de los tutores en campus.
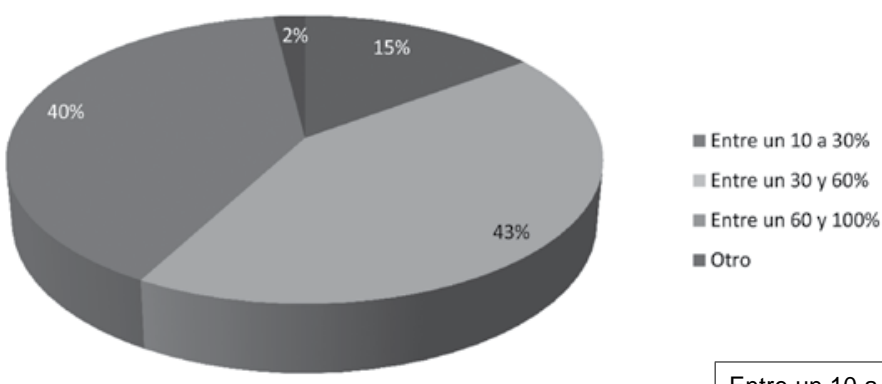

\begin{tabular}{|l|l|}
\hline $\begin{array}{l}\text { Entre un } 10 \text { a } \\
30 \%\end{array}$ & $15 \%$ \\
\hline $\begin{array}{l}\text { Entre un } 30 \text { y } \\
60 \%\end{array}$ & $43 \%$ \\
\hline $\begin{array}{l}\text { Entre un } 60 \text { y } \\
100 \%\end{array}$ & $40 \%$ \\
\hline Otro & $2 \%$ \\
\hline
\end{tabular}

Figura 4. El $43 \%$ afirma que solo entre un 30 y $60 \%$ de tutores de campus le hace falta más apoyo, motivación al estudiante, el $40 \%$ dice que entre un 60 y $100 \%$ les hace falta compromiso y dedicación en campus, un $2 \%$ comenta que Creería que un $90 \%$ de los tutores no apoya de la forma ideal como debería ser, se demora mucho para solucionar problemas si es que lo hace. 


\section{Revista de}

La figura 5 muestra los porcentajes sobre si el estudiante virtual tiene la capacidad de automotivarse y autodisciplinarse.

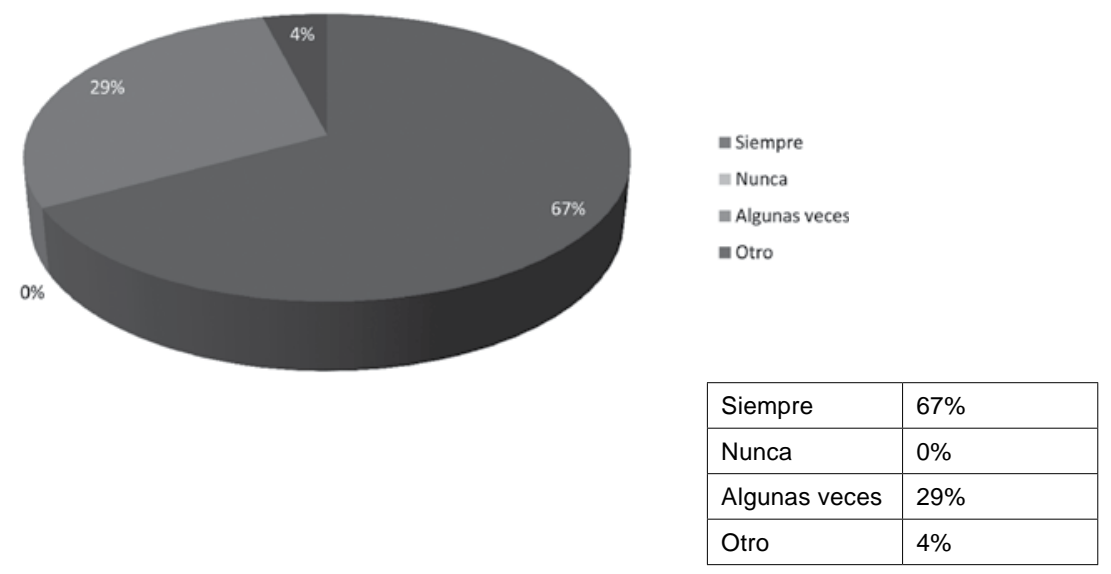

Figura 5. El $67 \%$ de los estudiantes dice que se auto motiva y autodisciplina en su proceso, el $29 \%$ algunas veces, el $4 \%$ dice que el tutor es parte importante en esa gestión para motivar al estudiante, sin embargo algunos lo hacen.

La figura 6 muestra los porcentajes sobre la realización de quiz con algún compañero de curso a su lado.

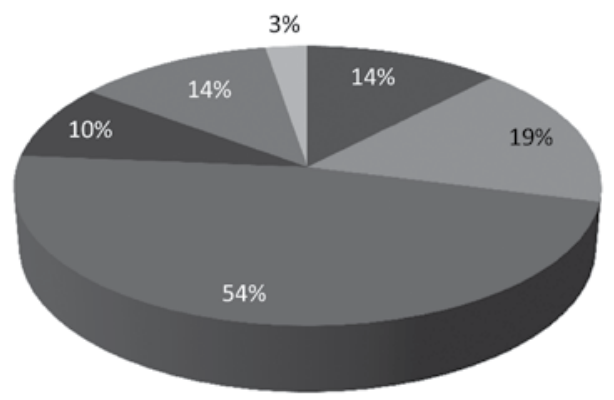

\begin{tabular}{|l|l|}
\hline Siempre & $14 \%$ \\
\hline Algunas veces & $19 \%$ \\
\hline Nunca & $54 \%$ \\
\hline $\begin{array}{l}\text { Cuando se } \\
\text { presenta la } \\
\text { eventualidad }\end{array}$ & $10 \%$ \\
\hline $\begin{array}{l}\text { No me parece } \\
\text { una conducta } \\
\text { ética }\end{array}$ & $14 \%$ \\
\hline Otro & $3 \%$ \\
\hline
\end{tabular}

Figura 6. El 54\% dice que nunca ha realizado algún quiz acompañado, el 19\% dice que algunas veces lo hace, el $14 \%$ dice que no es ético, el $10 \%$ comenta que cuando se presenta la eventualidad lo hace y el $3 \%$ dice que muy pocas veces lo hace, pero que si lo ha hecho, por chat, al lado del compañero. 


\section{Fabián José Mendoza Stave}

Análisis del desempeño virtual del rol de estudiantes de Ecacen, desde la perspectiva del desempeño del tutor como mediador del proceso, artículo producto de la investigación

La figura 7 muestra los porcentajes sobre la realización de alguna actividad evaluativa con algún compañero de curso

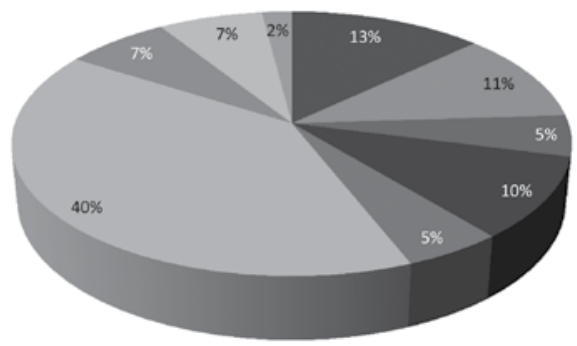

\begin{tabular}{|l|l|}
\hline Trabajar en equipo & $13 \%$ \\
\hline $\begin{array}{l}\text { Entre dos o varios } \\
\text { responden mejor }\end{array}$ & $11 \%$ \\
\hline Compañerismo & $5 \%$ \\
\hline Aprendizaje significativo en compañía & $10 \%$ \\
\hline Para obtener mejores calificaciones & $5 \%$ \\
\hline Nunca lo hecho & $40 \%$ \\
\hline $\begin{array}{l}\text { El sistema de educación a distancia lo } \\
\text { permite }\end{array}$ & $7 \%$ \\
\hline El aprendizaje virtual lo permite & $7 \%$ \\
\hline Otro & $2 \%$ \\
\hline
\end{tabular}

Figura 7. El $40 \%$ afirma que nunca lo ha hecho, el $13 \%$ afirma que lo hace para trabajar en equipo, el $11 \%$ dice que entre varios se puede responder mejor, el $10 \%$ dice que se hace aprendizaje significativo en compañía, el $7 \%$ dice que el sistema de EAD lo permite, $2 \%$ afirma que no les gusta pero muchos compañeros los convencen.

La figura 8 muestra sobre el conocimiento de las políticas de Netiqueta en AVA

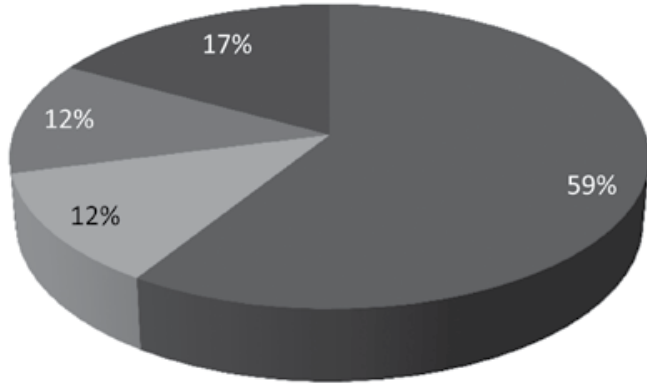

口i

No

No que es

- Debieran hablarle al estudiante 


\section{Revista de}

\begin{tabular}{|l|l|}
\hline Si & $59 \%$ \\
\hline No & $12 \%$ \\
\hline No que es & $12 \%$ \\
\hline $\begin{array}{l}\text { Debieran hablarle al } \\
\text { estudiante }\end{array}$ & $17 \%$ \\
\hline
\end{tabular}

Figura 8. El 59\% afirma que conoce las políticas de Netiquetas de campus de la Unad, el $17 \%$ dice que debería hacerle más comunicativo al estudiante las Netiquetas, el $12 \%$ no sabe que son Netiquetas y el $24 \%$ afirma que no sabe que es Netiquetas.

\section{La figura 9 muestra los porcentajes sobre la razón, porque no hay colaboración} en equipo.

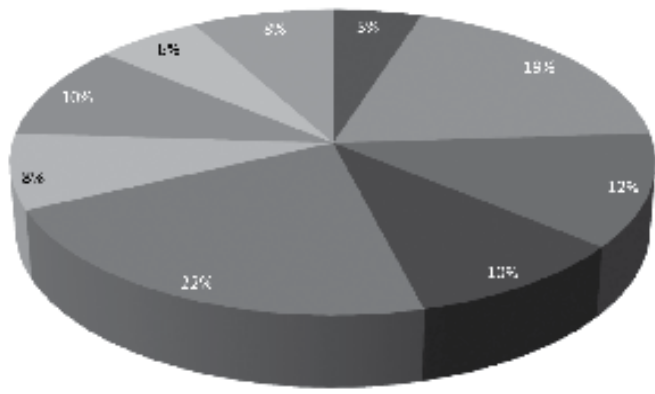

\begin{tabular}{|l|l|}
\hline Motivación del tutor & $5 \%$ \\
\hline Motivos personales del estudiante & $19 \%$ \\
\hline No les gusta el trabajo colaborativo & $12 \%$ \\
\hline Prefieren enviar el trabajo y no trabajar en equipo & $10 \%$ \\
\hline Todos dejan a última hora para enviar & $22 \%$ \\
\hline El sistema debe permitir el trabajo individual lo que interesa es cumplir el objetivo & $8 \%$ \\
\hline Concientizar a los compañeros sobre el trabajo en equipo & $10 \%$ \\
\hline El tutor nunca contesta mensajes en el foro colaborativo, no participa solo califica & $6 \%$ \\
\hline El tutor debe estar pendiente de cada mensaje para hacer seguimiento al proceso & $8 \%$ \\
\hline Otro & \\
\hline
\end{tabular}

Figura 9. El $22 \%$ afirma que todos los compañeros dejan a ultima hora el trabajo para enviar, el $19 \%$ afirma que no los envía por motivos personales, el $12 \%$ afirma que a sus compañeros no les gusta trabajar colaborativamente, el $10 \%$ dice que se debe concientizar a los estudiantes sobre el trabajo en equipo, el $8 \%$ dice que el tutor debe estar atento al foro para realimentar el trabajo, el $6 \%$ dice que el tutor nunca entra al foro, solo lo hace para calificar, el $5 \%$ dice que el tutor no motiva para el trabajo colaborativo. 


\section{Fabián José Mendoza Stave}

Análisis del desempeño virtual del rol de estudiantes de Ecacen, desde la perspectiva del desempeño del tutor como mediador del proceso, artículo producto de la investigación

La figura 10 muestra los porcentajes sobre si los tutores son los actores que le hace falta motivación para con los estudiantes para el trabajo colaborativo.

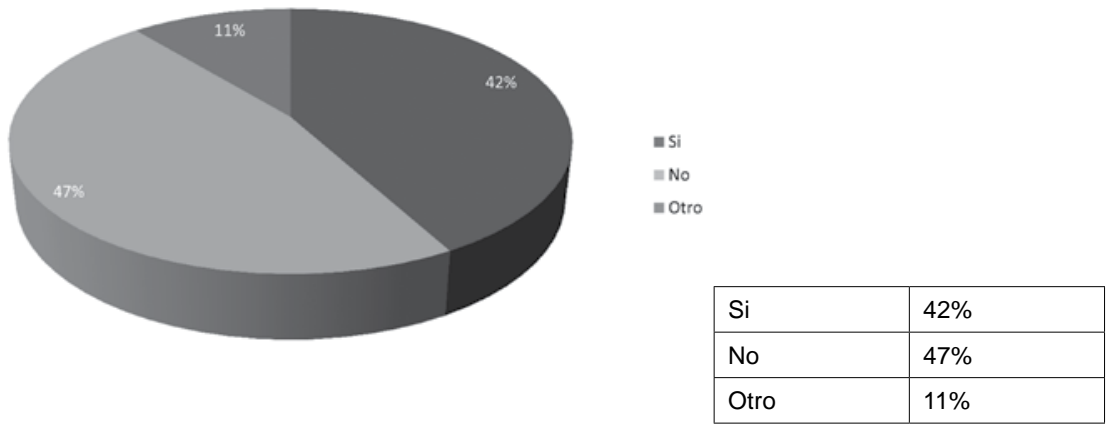

Figura 10. El $47 \%$ afirma que los tutores no son los actores de motivación para trabajar en equipo, se deduce que son los mismo estudiantes, el $42 \%$ afirma que los tutores deben motivar para el proceso del trabajo colaborativo, el $11 \%$ afirma que algunas veces, pero que siempre debe estar el tutor allí y que conteste los mensajes a tiempo.

La figura 11 muestra los porcentajes sobre cuáles serían las causales de fallas por parte del tutor virtual en el proceso de aprendizaje.

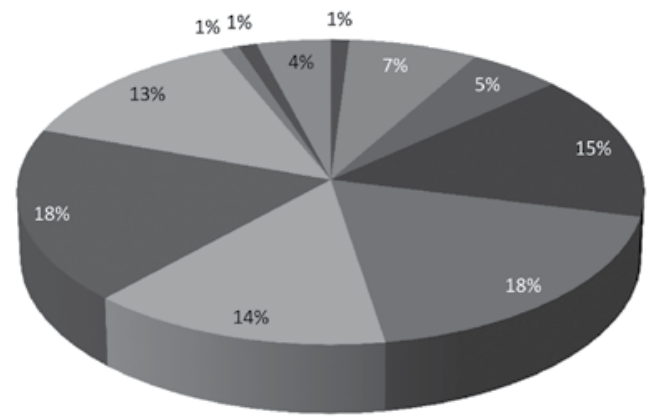

\begin{tabular}{|l|l|}
\hline Manejo de campus & $1 \%$ \\
\hline Sentido de pertenencia & $7 \%$ \\
\hline No hay fallas, excelentes tutores & $5 \%$ \\
\hline Oportunidad en la respuesta & $15 \%$ \\
\hline Retroalimentación inadecuada & $18 \%$ \\
\hline No hay motivación para el estudiante & $14 \%$ \\
\hline Entra pocas veces al foro a acompañar el trabajo colaborativo & $18 \%$ \\
\hline Demora para contestar los mensajes internos & $13 \%$ \\
\hline Pareciera que no conociera el tema tratado & $1 \%$ \\
\hline Demuestra baja calidad educativa & $1 \%$ \\
\hline
\end{tabular}




\begin{tabular}{|l|l|}
\hline La atención virtual es pésima & $4 \%$ \\
\hline Otro & \\
\hline
\end{tabular}

Figura 11. El $18 \%$ nos comenta que el tutor entra pocas veces al foro para acompañar el trabajo colaborativo, el $18 \%$ afirma que la retroalimentación no son las más adecuada, el $15 \%$ exige oportunidad en las respuestas, el $14 \%$ afirma que el tutor no motiva al estudiante durante el proceso, el $13 \%$ afirma que el tutor demora para contestar los mensajes internos, el $7 \%$ dice que no tiene sentido de pertenencia, el $5 \%$ dice que los tutores son excelentes, el $4 \%$ dice que la atención virtual es pésima, El $4 \%$ dice que algunos tutores califican demorado, que tienen muchos a cargo, el $1 \%$ demuestra baja calidad educativa.

La figura 12 muestra los porcentajes sobre los problemas que se consideran más graves en campus virtual con respecto al desarrollo del proceso de aprendizaje.

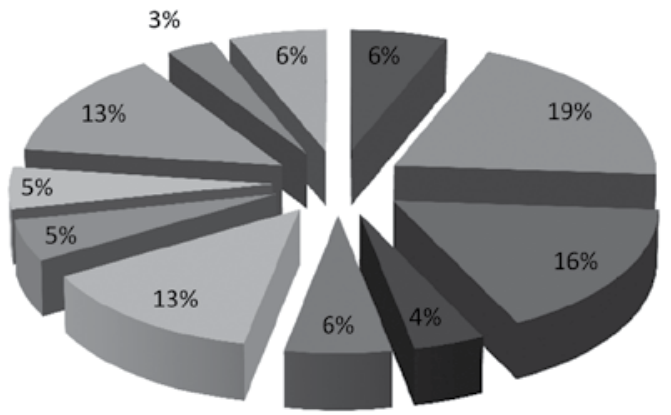

\begin{tabular}{|l|l|}
\hline Manejo de campus & $1 \%$ \\
\hline Sentido de pertenencia & $7 \%$ \\
\hline No hay fallas, excelentes tutores & $5 \%$ \\
\hline Oportunidad en la respuesta & $15 \%$ \\
\hline Retroalimentación inadecuada & $18 \%$ \\
\hline No hay motivación para el estudiante & $14 \%$ \\
\hline Entra pocas veces al foro a acompañar el trabajo colaborativo & $18 \%$ \\
\hline Demora para contestar los mensajes internos & $13 \%$ \\
\hline Pareciera que no conociera el tema tratado & $1 \%$ \\
\hline Demuestra baja calidad educativa & $1 \%$ \\
\hline La atención virtual es pésima & $4 \%$ \\
\hline
\end{tabular}

Figura 12. El 19\% dice que es bajo sentido de pertenencia del estudiante, el 16\% dice que baja motivación por parte de los actores, el $13 \%$ dice que hay bajo nivel ético por parte de los estudiante y que la plataforma de la unad no soporta los estudiantes, el $11 \%$ dice que deberían dejar abierta todas las actividades, el $6 \%$ dice que los cursos virtuales tienen bajo nivel académico, el $5 \%$ dice que la agenda muy rígida y que la interfaz al usuario en campus es muy difícil, el $4 \%$ dice que hay facilidad en los procesos académicos, el $3 \%$ dice que debería dividir el semestre y los cursos en trimestres. 


\section{Conclusiones}

Las propuestas de trabajos de investigación se derivan, por un lado, de las necesidades emergentes de los actores y, por otro, de los resultados de los estudios y aplicaciones institucionales. Es esperado que se examinen y aprovechan las aportaciones que realizan quienes a partir de la observación de la realidad y la reflexión profunda, aportan elementos teóricos para desprender nuevas perspectivas sobre los efectos de los medios en el aprendizaje y se recuperan las contribuciones de quienes, desde la praxis, buscan y encuentran posibilidades y beneficios en la incorporación de los recursos de la tecnología al proceso. El resultado obtenido se reflejaría en propósitos, planes que incorporan los avances teóricos para la fundamentación y la orientación de las acciones con base en la experiencia.

Los materiales educativos electrónicos basados en realidad virtual obligan a replantearnos el desarrollo profesional del profesor, del estudiante y de la actividad pedagógica que se genera. La mediación pedagógica virtual establece un tipo de dirección de aprendizaje que no es ni directa, todo lo contrario: indirecta y con la intervención activa de los involucrados en el proceso al propiciar la interacción y la interactividad de los estudiantes en este caso, el campus virtual.

Mientras que en la educación presencial tradicional el profesor concurre a las preguntas del grupo como la única manera de hacer participar y se esfuerza porque entiendan lo que se expone, en los nuevos ambientes de aprendizaje la mediación pedagógica permite un rango más amplio de formas de participación de los alumnos en clase. De ahí que una condición necesaria, aunque no suficiente, para que realmente haya participación de los estudiantes en el proceso de aprendizaje es la mediación pedagógica. Sin ella no es posible la interacción ni la interactividad y por consiguiente un aprendizaje significativo y que a mediano plazo será constructivista y se trabaja con sentido.

Internet rompe las barreras del tiempo y el espacio para desarrollar las actividades de enseñanza y aprendizaje, como se evidencia en las encuestas admite extender los estudios y formación a personas que por distintos motivos no pueden acceder a las aulas convencionales. Además la red rompe con el monopolio del docente como fuente principal del conocimiento y el proceso de aprendizaje no puede consistir en la sola recepción y memorización de datos recibidos en la clase, sino la permanente búsqueda, análisis y elaboración de informaciones obtenidas en las redes.

Hasta ahora, ninguno de los modelos diseñados para la educación a distancia ha sido descartado en la práctica, siguen vigentes. Sin embargo, es importante el predominio en la aplicación de las propuestas construidas alrededor de la tecnología, que la incorporan como vehículo y medio reforzador para el 
proceso de aprendizaje y que tratan de aprovechar las oportunidades que ofrecen las redes telemáticas y la multimedia, inimaginables por su riqueza, para la comunicación y la construcción de conocimiento. Lo anterior nos obliga a considerar las diferencias de los alumnos desde el punto de vista de la motivación (extrínseca e intrínseca), desde canales de percepción (visual, auditivo o); desde los estilos cognitivos (activo, reflexivo, teórico. Además, un grupo de habilidades y actitudes básicas personales para aprender en la distancia: autodisciplina, la capacidad de trabajar solo, la gestión del tiempo, cierto grado de independencia en el aprendizaje, la capacidad de realizar una programación personal compaginando vida personal, profesional y estudios.

La clave del cambio metodológico no es para aprender más, sino aprender diferente. El e-learning es un modelo de aprendizaje en el que el estudiante tiene que desarrollar habilidades tan importantes para su vida futura en esta sociedad.

Esta investigación arroja resultados muy alentadores que contribuyen a seguir avanzando en estos procesos de aprendizaje, si bien es cierto, según los estudiantes encuestados hay fallas en el proceso de acompañamiento que redundan de una u otra manera en la institución, muchas veces por sobre carga en estos que repercute de manera directa en su disposición académica en campus trayendo como consecuencia la desmotivación de los actores.

Cierto grado de estudiante, aquel que por sus condiciones y características personales le impiden ir a cursos tradicionales, hablan y se motivan intrínsecamente para realizar los cursos en campus, es un porcentaje bueno y promisorio, pero igualmente, muchas veces estos últimos se sienten afectados por el acompañamiento de algún o alguno s tutores que manifiestan ellos en un $60 \%$ no tener sentido de pertenencia, motivación por el acompañamiento.

Cabe resaltar que la mayoría de estos estudiantes están en eventos o períodos académicos avanzados, es decir, conocen el deber ser de lo académico, de las políticas de la universidad, de las mediaciones que se utilizan para adelantar estos procesos. Existe una falencia muy particular y es la honestidad de los estudiantes en compartir trabajos, quices y lecciones, con el propósito según ellos de compartir conocimientos, si bien es cierto, la metodología lo permite, pero sabemos que la edad de ellos, el evento académico cursado, permite inferir que tratamos con personas adultas con capacidad de discernir entre la ética de la convicción y lo malévolo del asunto. Partiendo siempre del sentido de la universidad que es al aprendizaje autónomo, basados en ayudas o mediaciones tecnológicas, iniciando desde lo moral, y el tipo de aprendizaje se puede colegir que es totalmente inaceptable este tipo de conductas. Aunque ellos están de acuerdo con que el aprendizaje en ambientes virtuales requiere de una conducta provista de honestidad y de principios éticos asociados a una voluntad y deseo de superación personal. 
Análisis del desempeño virtual del rol de estudiantes de Ecacen, desde la perspectiva del desempeño del tutor como mediador del proceso, artículo producto de la investigación

Se puede colegir también que el desempeño de los tutores no es el más adecuado con respecto al tipo de mediación, en la mediación tradicional encuentran apoyo, se dice que porque los tienen allí y se siente el calor humano, pero que en la parte virtual solo alguno de ellos muestra preocupación, motivación y sentido de pertenencia sobre los cursos que son responsables, sin embargo, muchos de ellos si acompañan el proceso como debe ser.

\section{Referencias bibliográficas}

Arriaga, J. Determinación de un modelo causal de los factores de calidad docente en entornos virtuales de aprendizaje, Dirección General de Universidades, Secretaría de Estado de Universidades e Investigación Ministerio de Educación y Ciencia, Universidad Politécnica de Madrid. 2005

Cookson, Peter. Los ambientes de aprendizaje en la educación a distancia, en: Desarrollo de ambientes de aprendizaje en educación a distancia. CECAD. Mexico, Universidad de Guadalajara, 2008.

Duart, J. M. (31 de octubre de 2001). Evaluación de la calidad docente en entornos virtuales de aprendizaje. Recuperado el 19 de junio de 2009, de www.uoc.edu/web/: http://reddigital.cnice.mec.es/6/Documentos/docs/articulo12_material.pdf

Guitert, M; Romeo, T; Perez-Mateo, M. Competencias TIC y trabajo en equipo en entornos virtuales, Revista Universitaria de la Sociedad y el Conocimiento (RUSC), vol. $4 \mathrm{~N}^{\circ}$ 1, p.1-12. (2007)

Moreno, Castañeda Manuel. Desarrollo de ambientes de aprendizaje en educación a distancia. Coordinación de Educación Continua Abierta y a Distancia de la Universidad de Guadalajara. México, Guadalajara, 2008

Serrano, A; Martínez, E. La brecha digital. Mitos y realidades, Baja California, México, Departamento Editorial Universitaria Universidad Autónoma Baja California. 2008. 